\title{
RADIAL DISTRIBUTION FUNCTIONS AND COMPRESSIBILITY FACTORS FOR BINARY MIXTURE OF HARD SPHERES FROM MOLECULAR DYNAMICS SIMULATION
}

\author{
B. KUROCZYCKI, M. BANASZAK, AND S. JURGA \\ Institute of Physics, A. Mickiewicz University, Umultowska 85, 61-614 Poznań, Poland
}

(Rec. 26 October 2004)

\begin{abstract}
Molecular dynamics simulation of binary mixtures of hard spheres with large size ratio is reported. Radial distribution functions, their contact values and the compressibility factors are recorded at three state points. A reasonably good agreement with theory and Monte Carlo simulations is shown.
\end{abstract}

\section{INTRODUCTION}

Computer simulations of hard sphere mixtures can significantly help in understanding the physical properties of a variety of molecular systems, such as, for example, colloidal suspensions. Hard-sphere systems were the first molecular models investigated by computer simulations, starting with the classical Metropolis Monte Carlo (MC) study [1] of 32 hard spheres placed in a cubic box with the standard periodic boundary conditions. Molecular dynamics (MD) simulations followed, resulting in new and sometimes quite unexpected results. For example, one of the first interesting findings obtained from hard-sphere simulations was the well-defined freezing transition demonstrated in 1959 by Alder and Wainwright [2], and Wood and Jacobson [3]. At that time it was believed that only the attractive forces can drive a freezing transition in simple fluids. The results were debatable for a long time. and currently it is accepted that identical hard spheres systems have a fluid-solid transition. The freezing transition can be accounted for by entropy considerations, i.e., the entropy of crystalline phase is higher than entropy of fluid phase at sufficiently high densities [4].

The binary hard-sphere mixture serves as a model for mixtures of colloids and polymers, or other colloidal systems. This binary fluid mixture of large and small hard spheres is not miscible for all size ratios and compositions as discussed in a review paper by Dijkstra et al. [4]. The full phase diagram of this fluid is still debated. (The general mechanism of phase separation in hard-body systems, known as entropic phase separation, is discussed in detail in references [5-7]). If the system, however, does not phase-separate then it can be considered as a model for stable colloidal suspension, with the large hard spheres being the colloid particles, and the small spheres being the solvent molecules. For colloidal suspensions the typical size ratio, $R=d_{11} / d_{22}$, is much bigger than unity, where $d_{22}$ is the diameter of the smaller hard sphere, and $d_{11}$ is the diameter of the larger hard sphere. To simulate colloidal suspension 
the Brownian Dynamics (BD) method is often used. In this type of simulation only the larger molecules are considered explicitly, and the presence of the solvent is introduced via an effective background which gives rise to friction and thermal noise. The drawback of the Brownian Dynamics is the fact that the hydrodynamic interactions are not taken into account. Sometimes the hydrodynamic interactions are approximated by using the Onseen tensor, but this is based on analytical approximations which are difficult to control.

Phase behavior of the simple fluid is similar to the phase behavior of a colloidal suspension. Similarly, the static and dynamics properties of colloidal suspension are similar to those of the of simple fluid. However, the diffusivities and relaxation times for fluid molecules are about $10^{9}$ larger than relaxation times of molecules in suspensions. Therefore, lifetime of the metastable phase of suspension observed before crystallization, can be long enough (from seconds to hours) to allow experimental studies. This long time gives possibility to accurately measure physical properties in this state [8]. Colloidal suspension can also be studied by many experimental methods such as rheological experiments (viscosity and elastic properties) [8], light scattering (structural properties and diffusion coefficients) [9] and nuclear magnetic resonance.

In this work, we perform the MD simulation of binary hard spheres mixture with a large size ratio. Hydrodynamic interactions between larger spheres (mediated by small spheres) are taken into account explicitly in this model, and no approximation, such as the Onseen tensor, are used. The goal of this paper is to perform a preliminary molecular dynamics study of binary mixtures of hard spheres in order to calculate the compressibility factors, Z, from the radial distribution functions and to compare the results of this calculation to the results of the corresponding Monte Carlo simulations [10] and theoretical predictions. In particular, we compare our results with the results of Boublik-Mansoori-Carnahan-Starling-Leland (BMCSL) theory [11, 12], the results of Henderson-Chan (HC) theory [13, 14], the results of Percus-Yevick (SYH) theory [15], the results of Matyushov-Ladanyi (ML) theory [16], and results of the MC simulation [10].

The following preliminary MD study serves merely as an introduction to a future work which is intended to focus on dynamical properties of colloidal suspensions, such as diffusion, and which is to be carried out with an optimized parallel MD code.

\section{MODEL AND SIMULATION METHOD}

In this paper we simulate a binary hard sphere mixture, i.e., a system consisting of many small spheres (of type 2) surrounding a few large spheres (of type 1) as shown in Fig. 1. This system is characterized by volume fractions $x_{1}$ and $x_{2}$, defined as follows

$$
x_{i}=\frac{N_{i}}{N_{\text {total }}} \text {, }
$$


where $N_{\text {total }}$ is the total number of spheres, and is the number of spheres of type $i$ ( $i=1$ for large spheres, and $i=2$ for small spheres). The number density is defined as

$$
\rho=\frac{N_{\text {total }}}{V}
$$

where $V$ is volume, and the reduced density can be expressed as

$$
\rho^{*}=\rho\left(x_{2} d_{22}^{3}+x_{1} d_{11}^{3}\right)
$$

Finally the size ratio $R$ is

$$
R=\frac{d_{11}}{d_{22}},
$$

where $d_{11}$ is the diameter of a large sphere, and $d_{22}$ is the diameter of a small sphere.

Fig. 1. A snapshot of typical configuration of binary hard sphere mixture $(R=5$; $\left.N=9637 ; \rho^{*}=0.6 ; x_{2}=0.00311\right)$

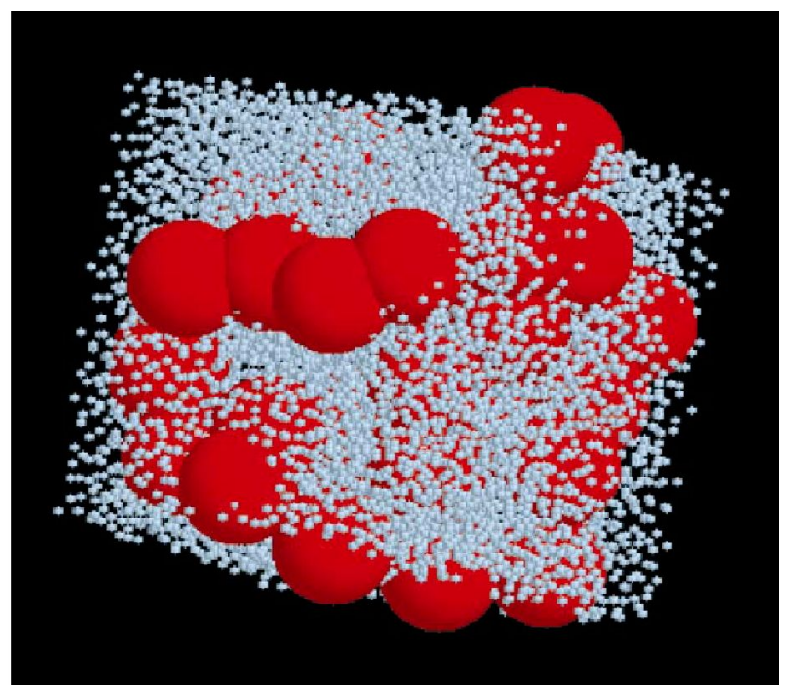

The MD simulation of the binary mixture of hard spheres is performed by the standard algorithm [17, 18], i.e., the molecules move freely between collisions, and most of the effort is directed towards predicting and executing collisions. The small spheres are initially placed on a 3D cubic lattice in order to enable dense packing fractions. The large spheres, on the other hand, assume random initial positions. The velocities for the spheres are draw from a uniform angular distribution. Once the initial conditions are set, and the cubic periodic boundary conditions imposed, the system can follow its trajectory with the precision of the computer hardware (20 Intel-based-processor cluster). The MD time step is defined as a time interval between successive collisions. The simulation is run for $100 \times 10^{6}$ MD time steps for each 
state point considered. Velocities and positions are recorded every $1 \times 10^{6}$ steps. The system is equilibrated for $50 \times 10^{6}$ steps, and then the properties of interest are calculated from the equilibrated configurations. We calculate the radial distribution functions (rdf) using the standard method [19], and report the radial distribution functions, $g_{i j}(r)$, where $i=1$ or 2 , with 1 and 2 corresponding to the large and small spheres, respectively. The diameter of the small sphere, $d_{22}$ is chosen as a length unit, and we also define $d_{12}$ as $d_{12}=\left(d_{11}+d_{22}\right) / 2$.

The compressibility factor, $Z$, is defined as

$$
Z=\frac{P}{\rho k_{\mathrm{B}} T},
$$

where $P$ is the pressure of the system, $T$ is temperature, and $k_{\mathrm{B}}$ is the Boltzmann constant. For hard-sphere binary system, the compressibility factor depends only on the reduced density and composition (does not depend on temperature), and can be expressed in terms of the contact values of the radial distribution functions, $g_{i j}\left(d_{i j}\right)$ as

$$
Z=1+\frac{2 \pi \rho}{3} \sum_{i j} x_{i} x_{j} d_{i j}^{3} g_{i j}\left(d_{i j}\right)
$$

or in more detail as

$$
Z=1+\frac{2 \pi \rho}{3}\left(x_{1}^{2} d_{11}^{3} g_{11}\left(d_{11}\right)+x_{2}^{2} d_{22}^{3} g_{22}\left(d_{22}\right)+2 x_{1} x_{2} d_{12}^{3} g_{12}\left(d_{12}\right)\right)
$$

\section{RESULTS AND DISCUSSION}

The simulation is carried out for three state points at the following conditions (the same as in Ref. [10]):
A) $R=5 ; 1=9637 ; \rho^{*}=0.6 ; x_{2}=0.00311$.
B) $R=5 ; \quad 8000 ; \rho^{*}=0.6 ; x_{2}=0.005$.
C) $R=5 ; 1=6803 ; \rho^{*}=0.658 ; x_{2}=0.00588$.

For every state point we calculate contact values of the radial distribution functions $\left(g_{11}\left(d_{11}\right),{ }_{g 12}\left(d_{12}\right), g_{22}\left(d_{22}\right)\right)$ and the corresponding compressibility factors (pressures) which are compared to results from other sources [10]. All results and comparisons are summarized in Figs. 2, 3 and 4, and Tabs. 1, 2, and 3. The results from simulations and theory are presented in Tabs. 1, 2, and 3, where we use the following labels:

HS - results from Molecular Dynamics obtained from our simulation,

MC - results from Monte Carlo simulation [10],

BMCSL - results from Boublik-Mansoori-Carnahan-Starling-Leland theory [11, 12],

HC - results from Henderson-Chan theory [13, 14],

SYH - results from Percus-Yevick theory,

ML - results from Matyushov-Ladanyi theory [16]. 

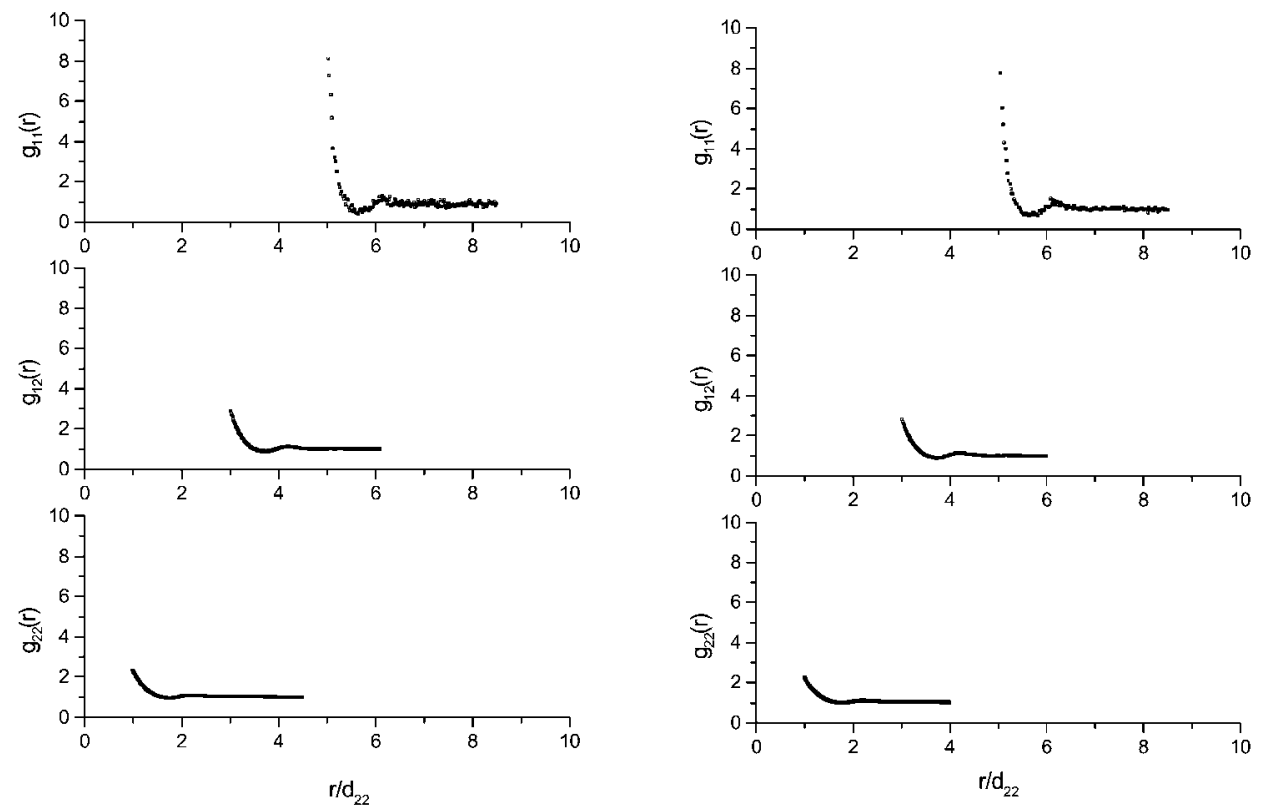

Fig. 2. Radial distribution functions for the first system (A); from top to bottom: $\mathrm{g}_{11}(r)$, $g_{12}(r)$, and $\mathrm{g}_{22}(r)$

Fig. 3. Radial distribution functions for the second system (B); from top to bottom: $g_{11}(r)$, $g_{12}(r)$, and $g_{22}(r)$

Fig. 4. Radial distribution functions for the third system (C); from top to bottom: $g_{11}(r)$, $g_{12}(r)$, and $g_{22}(r)$
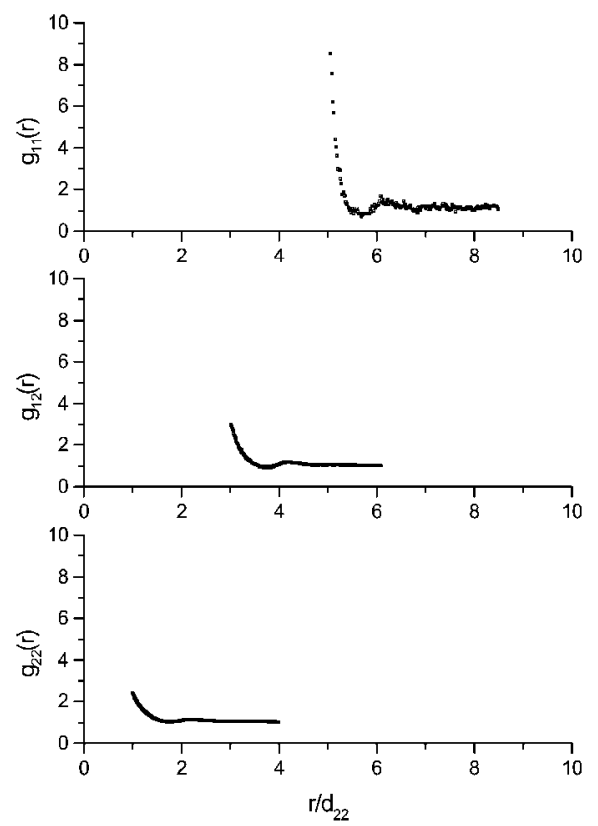
Table 1. Contact values for the radial distribution functions and the compressibility factor for the first system (A)

\begin{tabular}{lcccl}
\hline & $g_{11}\left(d_{11}\right)$ & $g_{12}\left(d_{12}\right)$ & $g_{22}\left(d_{22}\right)$ & $Z$ \\
\hline HS & 8.3 & 2.918 & 2.317 & 3.538 \\
MC & 10.21 & 3.126 & 2.337 & 3.592 \\
BMSCL & 7.643 & 3.009 & 2.327 & 3.562 \\
HC & 19.482 & 3.106 & 2.327 & 3.59 \\
SYH & 5.936 & 2.951 & 2.354 & 3.576 \\
ML & & 3 & & \\
\hline
\end{tabular}

Table 2. Contact values for the radial distribution functions and the compressibility factor for the second system (B)

\begin{tabular}{lcccc}
\hline & $g_{11}\left(d_{11}\right)$ & $g_{12}\left(d_{12}\right)$ & $g_{22}\left(d_{22}\right)$ & $Z$ \\
\hline HS & 7.567 & 2.790 & 2.199 & 3.288 \\
MC & 9.137 & 2.85 & 2.235 & 3.332 \\
BMSCL & 6.749 & 2.816 & 2.224 & 3.311 \\
HC & 13.333 & 2.895 & 2.224 & 3.343 \\
SYH & 5.45 & 2.789 & 2.256 & 3.327 \\
ML & & 2.748 & & \\
\hline
\end{tabular}

Table 3. Contact values for the radial distribution functions and the compressibility factor the third system $(\mathrm{C})$

\begin{tabular}{lclll}
\hline & $g_{11}\left(d_{11}\right)$ & $g_{12}\left(d_{13}\right)$ & $g_{22}\left(d_{22}\right)$ & $Z$ \\
\hline HS & 11.992 & 3.021 & 2.389 & 3.681 \\
MC & 11.37 & 3.127 & 2.414 & 3.727 \\
BMSCL & 7.781 & 3.102 & 2.41 & 3.704 \\
HC & 17.773 & 3.2 & 2.241 & 3.764 \\
SYH & 6.19 & 3.08 & 2.459 & 3.733 \\
ML & & 3.072 & & \\
\hline
\end{tabular}

In Figs. 2, 3 and 4 we report the radial distribution functions from which we can extract their contact values at the state points considered. Comparison our data with the other results shows that they do not differ significantly, e.g. the difference for the compressibility factor is less than $3 \%$. 


\section{CONCLUSION}

Molecular dynamics simulations of binary hard-sphere mixtures are in a reasonably good agreement with theory and Monte Carlo simulations. This simulation verifies the $\mathrm{C} / \mathrm{C}^{++}$source code developed for this research project. This MD study is an introduction to a future work which is intended to focus on studies of dynamical properties of colloidal suspensions, such as diffusion, and which are to carried out with an optimized parallel MD code.

\section{References}

[1] N. Metropolis, A. W. Resenbluth, M. N. Rosenbluth, A. H. Teller, and E. Teller, J. Chem. Phys, 21, 1087 (1953).

[2] B. J. Adler and T. E. Wainwright, J. Chem. Phys. 27, 1208 (1957).

[3] W. W. Wood and J. D. Jacobson, J. Chem. Phys. 27, 1207 (1957).

[4] M. Dijkstra, R. van Roij, and R. Evans, Phys. Rev. E, 59, 5744 (1999).

[5] H. N. W. Lekkerkerker and A. Stroobants, Physica A, 195, 387 (1993).

[6] Y. Rosenfeld, Phys. Rev. Lett. 73, 3831 (1994).

[7] K. W. Wojciechowski, Physica A, 232, 723 (1996).

[8] T. G. Mason and D. A. Weitz, Phys. Rev. Lett. 75, 2770 (1995).

[9] P. N. Segre, S. P. Meeker, P. N. Pusey, and W. C. Poon, Phys. Rev. Lett. 75, 958 (1995).

[10] D. Cao, K.-Y. Chan, D. Henderson, and W. Wang, Molec. Phys. 98, 619 (2000).

[11] T. Boublik, J. Chem. Phys. 53, 471 (1970).

[12] G. A. Mansoori, N. F. Carnhan, K. E. Starling, and T. W. Leland, J. Chem. Phys. 54, 1523 (1971).

[13] D. Yau, K. Y. Chan, and D. Henderson, Molec. Phys. 88, 1237 (1996).

[14] D. Yau, K. Y. Chan, and D. Henderson, Molec. Phys. 91, 1137 (1997).

[15] D. Henderson, D. Sokolowski, and D. Wasan, Phys. Rev. E, 57, 5539 (1998).

[16] D. V. Matyushov and B. M. Ladanyi, J. Chem. Phys. 107, 5815 (1997).

[17] B. J. Alder and T. E. Wainwright, J. Chem. Phys. 31, 459 (1959).

[18] B. J. Alder and T. E. Wainwright, J. Chem. Phys. 33, 1439 (1960).

[19] M. P. Allen and D. J. Tildesley, Computer Simulation of Liquids, Oxford University Press, Oxford 1987. 